\title{
Posterior capsule opacification and Nd:YAG laser rates with two hydrophobic acrylic single-piece IOLs
}

\author{
Daniel Schartmüller ${ }^{1} \cdot$ Sabine Schriefl ${ }^{1}$ Luca Schwarzenbacher ${ }^{1} \cdot$ Christina Leydolt $^{1} \cdot{\text { Michael Kundi } \mathbb{C}^{2}}^{2}$ \\ Stephan Pieh ${ }^{1} \cdot$ Rupert Menapace ${ }^{1} \cdot$ Katharina Kriechbaum ${ }^{1}$
}

Received: 7 February 2019 / Revised: 26 July 2019 / Accepted: 7 August 2019 / Published online: 4 September 2019

(c) The Author(s), under exclusive licence to The Royal College of Ophthalmologists 2019

\begin{abstract}
Objectives To evaluate the development of posterior capsule opacification (PCO) and Nd:YAG capsulotomy rates following implantation of two hydrophobic acrylic IOLs.

Methods In a randomized, controlled trial, 80 patients with bilateral senile cataract were implanted with the hydrophobic acrylic single-piece intraocular Lenses (IOLs) EyeCee One in one eye and iMics1 in the other. Outcomes of 39 patients (78 eyes) were evaluated after 3 years. Automated Quantification of After-Cataract (AQUA; for PCO occurrence), visual acuity, anterior fibrosis, capsule-optic edge interaction and distance between anterior and posterior capsule IOL surface were analysed.

Results After a mean follow-up of $38 \pm 1.95$ months, Nd:YAG capsulotomy occurred at a rate of $15.4 \%$ and $46.2 \%$ in the EyeCee One and iMics1 groups, respectively $(p<0.01)$. Respective mean PCO scores measured by AQUA were $1.57 \pm 1.63$ and $2.45 \pm 1.44(p=0.019)$. A distinct gap between the anterior capsule and the IOL optic was present in $89 \%$ of eyes implanted with EyeCee One and 13\% of iMics1 eyes. A gap between the posterior capsule and the posterior surface of the lens was observed in 76\% of EyeCee One eyes and 35\% of iMics1 eyes.

Conclusions Study findings suggest that PCO and Nd:YAG capsulotomy rates are significantly lower in eyes implanted with the EyeCee One IOL compared to the iMics1 IOL. Optic sharpness and lens material seem to be the decisive factors, while the stepped edge beneath the haptic junction appeared to be ineffective.
\end{abstract}

\section{Introduction}

Posterior capsule opacification (PCO) remains the most common complication of cataract surgery with Neodymium:Yttrium-Aluminium-Garnet (Nd:YAG) capsulotomy used as an effective but damaging and expensive PCO treatment of limited availability. Improvements in surgical technique and modifications in IOL design and

Supplementary information The online version of this article (https:// doi.org/10.1038/s41433-019-0569-x) contains supplementary material, which is available to authorized users.

Rupert Menapace

rupert.menapace@meduniwien.ac.at

1 Department of Ophthalmology and Optometry of the Medical University of Vienna, Spitalgasse 23, 1090 Vienna, Austria

2 Center for Public Health, Medical University of Vienna, Kinderspitalgasse 15, 1090 Vienna, Austria material have all led to a decrease in PCO incidence. In recent years manufacturers came up with the so-called "enhanced posterior optic edge" IOLs featuring a continuous sharp optic edge. Opinions about the efficacy of the uninterrupted square optic edge differ among several studies. In recent studies evaluating IOLs with a continuous sharp optic-edge and interrupted sharp optic-edge, the authors did not find significant differences in PCO development after 3 years [1, 2]. In contrast to these results another study concluded that a $360^{\circ}$ square optic edge with its haptic offset causes tighter positioning of the posterior capsule against the posterior optic and may be an argument for lower PCO development compared to a discontinuous sharp optic edge [3].

This study was designed to look specifically at the PCO and Nd:YAG capsulotomy rates associated with two hydrophobic IOLs of hydrophobic acrylic material with different design elements: EyeCee One hydrophobic acrylic IOL-biplanar, continuous square optic edge (NS-60YG; Nidek Co. Ltd.) and iMics1 hydrophobic acrylic IOL 
Table 1 Characteristics of IOL $\mathrm{A}$ and IOL B

\begin{tabular}{|c|c|c|}
\hline & EyeCee One (IOL A) & iMics 1 (IOL B) \\
\hline Overall length & $13.0 \mathrm{~mm}$ & $12.5 \mathrm{~mm}$ \\
\hline Optic diameter & $6.0 \mathrm{~mm}$ & $6.0 \mathrm{~mm}$ \\
\hline Optic edge & $360^{\circ}$ sharp optic edge & $\begin{array}{l}\text { Sharp/ interrupted at the optic-haptic } \\
\text { junctions }\end{array}$ \\
\hline Material & $\begin{array}{l}\text { Copolymer of } n \text {-butyl acrylate, } n \text {-butyl } \\
\text { methacrylate and phenoxyethyl acrylate }\end{array}$ & $\begin{array}{l}\text { Copolymer of phenylethyl methacrylate and } \\
n \text {-butyl acrylate, fluoroalkyl methacrylate }\end{array}$ \\
\hline Haptics & C-loop & C-loop $5^{\circ}$ angulation \\
\hline Filter & UV- and Blue Light Filter & UV- and Blue Light Filter \\
\hline Incision size & $>2.2 \mathrm{~mm}$ & $>1.8 \mathrm{~mm}$ \\
\hline
\end{tabular}

-uniplanar, interrupted square optic edge (AF-1 NY-60; HOYA Surgical Optics, Inc.).

\section{Materials and methods}

This prospective, randomized controlled trial involved 80 patients with bilateral senile cataract implanted with the iMics1 IOL in one eye and the EyeCee One IOL in the contralateral eye. The study was conducted by the Department of Ophthalmology at the Medical University of Vienna, Austria. All enrolled patients provided written informed consent before participating in the study. Patients were operated by the same surgeon in both eyes. The study was performed in adherence to the tenets of the Declaration of Helsinki and with the approval from the Ethics Committee and Research Board of the study institution (EK 933/2011).

Inclusion criteria were bilateral age-related cataract, age of at least 40 years and an absence of medical eye history or physical examination findings that are considered as contraindications for cataract surgery. Exclusion criteria included significant additional ophthalmic diseases (such as pseudoexfoliation and progressed retinal degenerations), uncontrolled systemic diseases and history of ocular surgery, uveitis or trauma.

\section{The IOLs}

The study IOLs were the EyeCee One $=$ IOL A (NS-60YG; Nidek Co. Ltd., Japan) and the iMics1 = IOL B (NY-60; Hoya Surgical Optics, Inc., Japan)—one-piece hydrophobic acrylic IOLs with modified C-loop haptics, sharp edges, $6 \mathrm{~mm}$ optic diameter and a total diameter of $13 \mathrm{~mm}$ (IOL A) and $12.5 \mathrm{~mm}$ (IOL B). Table 1 shows the specific features for each IOL.

\section{Preoperative evaluation}

Before cataract surgery, all patients underwent visual acuity testing using a Snellen chart. Slit lamp examination and fundoscopy were also performed. Preoperative biometry was performed using the IOLMaster 500 (Zeiss Meditec AG, Jena, Germany). The SRK/T formula was used to determine IOL power.

\section{Surgical technique}

All patients were randomly allocated to receive an EyeCee One or an iMics1 IOL in their first eye and the other study IOL in their second eye. Both eyes underwent IOL implantation on the same day or within 2 weeks. All surgeries were performed by two surgeons (RM, SP) following manual standard phacoemulsification cataract surgery protocol. First, a temporal $2.2 \mathrm{~mm}$ self-sealing incision was made, the anterior chamber was filled with an ophthalmic viscoelastic device, and a continuous curvilinear capsulorhexis was created. Hydrodissection and phacoemulsification were then performed and followed by IOL implantation. The surgeon was unaware to which IOL type was implanted until the end of phacoemulsification. After IOL implantation, the remaining OVD was aspirated and residual lens fibres were removed from the posterior capsule. No anterior capsule polishing was performed. On the contrary, care was taken not to compromise the integrity of the anterior lens epithelial cell (LEC) layer on the posterior surface of the residual anterior capsule overlapping the optic in order not to interfere with the capsular fusion and fibrotic sealing process [4].

\section{Postoperative procedure}

Postoperative topical therapy included dexamethasone/ gentamycin (Ursapharm, Saarbrücken, Germany) three times daily for 3 weeks and bromfenac (Dr. Gerhard Mann, Chem.-pharm. Fabrik GmbH, Berlin, Germany) twice daily for 2 weeks. Postoperative follow-up was performed at 3 years, and involved visual acuity testing, slit lamp examination, fundoscopy, capsular bag performance evaluation and digital retroillumination photography.

Automated Quantification of After-Cataract (AQUA) analysis of the digital retroillumination images were used to objectively quantify PCO occurrence [5]. The AQUA analysis 
calculates a score on a scale of $0-10$ for each eye, where 0 was equivalent to the complete absence of PCO and 10 reflected severe PCO. Photos were attained using a highresolution digital retroillumination imaging system. A digital colour camera (KODAK DCS720x) was used to capture frontal images. Image analysis was performed using dedicated analysis software developed at the Institute for Computer Graphics and Vision (ICG) at Graz Technical University [57]. After outlining the capsulorhexis edge and identifying the region of interest, the software used a local co-occurrence matrix method to yield segmentation of the image. Visual acuity testing was performed with a Snellen chart and converted to the logarithm of the minimum angle of resolution (logMAR) for statistical analysis. Capsular bag performance was graded subjectively at the slitlamp. Anterior and posterior IOL optic-capsule distance, anterior fibrosis, anteriorposterior capsule interaction along the optic edge, posterior capsule fibrosis and subjective PCO morphology (monolayer, syncytium or pearls) were specified and graded. If there was a decrease of vision due to after-cataract, the patient received Nd:YAG laser capsulotomy treatment. Total Nd:YAG rates were evaluated. The presence of anterior fibrosis was subjectively graded during slitlamp analysis. Four different grades were achievable, ranging from 0 for no fibrosis to +++ for severe fibrosis. The presence or absence of a gap between the anterior capsule leaf and the anterior optic surface as well as that between the posterior capsule and the posterior optic surface was evaluated under oblique slitbeam illumination. The interaction between the anterior and the posterior capsule at the optic rim was subjectively classified to "P" (parallel), "Y" (Y shape), "R" (right angled) and "W" (wrapped) capsular bend configuration. In accordance with the classification developed by Sacu et al., capsular bending was graded typically along the optic circumference between the haptic insertions, at least 1 clock hour at a distance to the junction [8].

\section{Statistical analysis}

Results are presented as means and standard deviation (SD). Statistical analyses were performed using IBM SPSS Statistics versions 23.0.0.3 (IBM Corp., NY, USA). If one eye in a patient had to be excluded due to a small pupil diameter or previous Nd:YAG laser capsulotomy, the other eye was also excluded from statistical analyses. Continuous data were compared between IOL A and IOL B One using Wilcoxon's matched-pairs test, and categorical data were compared using McNemar's test. A $p$ value of less than 0.05 was considered statistically significant.

Sample size was selected to detect a difference in PCO percentage between groups of $5 \%$ after 3 years using an SD of about $15 \%$. To account for a dropout rate of about $55 \%$ after 3 years in this elderly population, 80 patients were included in the study.

\section{Results}

Of the 80 patients initially enrolled in the study, 78 eyes of 39 patients ( 12 men, 27 women) with a mean age of $70.84 \pm$ 7.88 participated in the study for the full 3-year follow-up. Death $(n=1)$, immobility $(n=15)$, resettlement $(n=1)$, patients discontinuation $(n=10)$ failure to present for follow-up examinations with an arranged appointment $(n=$ 3 ) and inability to contact via telephone or letter invitation $(n=11)$ were the primary reasons 41 patients from the original cohort were lost to follow-up. All 39 patients among the final cohort were followed up for a mean duration of $38 \pm 1.95$ months. Mean best corrected visual acuity (BCVA) was $0.032 \pm 0.053 \log$ Mar in the IOL A group and $0.089 \pm$ $0.11 \log$ MAR in the IOL B group $(n=29 ; p<0.01$; Wilcoxon). This corresponds to a mean Snellen visual acuity of about 0.94 for IOL A and 0.84 for IOL B, respectively. There were three patients $(7.7 \%)$ and ten patients $(25.6 \%)$ who received Nd:YAG laser capsulotomy treatment prior to the 3year follow-up in the IOL A and in the IOL B Group, respectively $(p=0.04 ;$ McNemar). Three-year Nd:YAG capsulotomy rates were $15.4 \%$ and $46.2 \%$ in the IOL A and IOL B groups, respectively ( $n=39 ; p<0.01 ;$ McNemar). As shown in Fig. 1, higher mean PCO scores, measured by AQUA, were observed among the IOL $\mathrm{B}$ group with respective scores of $1.57 \pm 1.63$ and $2.45 \pm 1.44(n=29 ; p=$ 0.019; Wilcoxon). The rhexis was on optic in all IOLs where rhexis overlap was evaluable $(n=36)$.

A gap was present between the anterior capsule and anterior optic lens surface in 33 eyes $(89.2 \%)$ implanted with IOL A and $5(12.8 \%)$ IOL B eyes $(n=37 ; p<0.01 ;$ McNemar). A gap was observed between the posterior capsule and the posterior surface of the lens in $22(75.9 \%)$ IOL A eyes and 10 (34.5\%) IOL B eyes $(n=29 ; p<0.01$; McNemar).

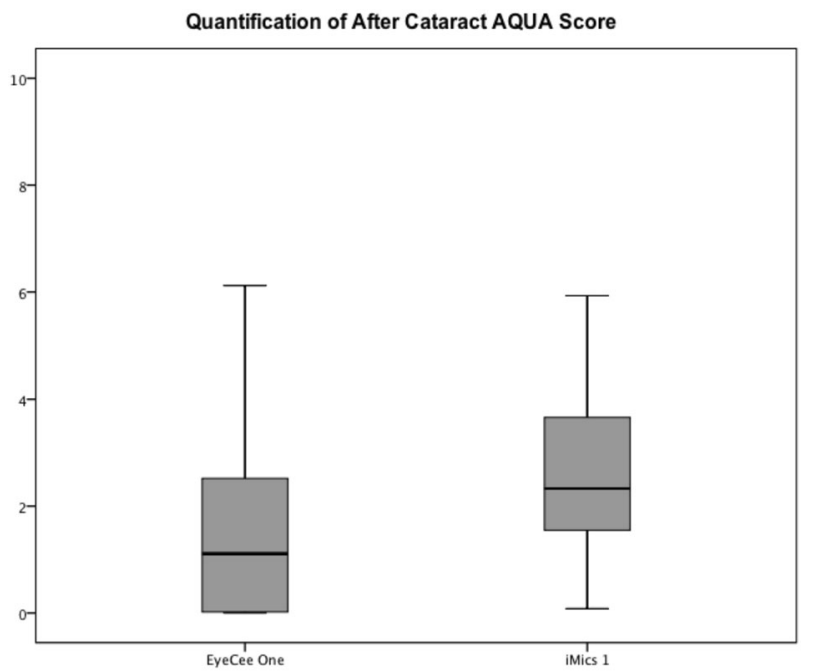

Fig. 1 PCO scores among both IOL groups, as measured by AQUA $p=0.019$ Wilcoxon. Centre value $=$ Median 
PCO subtype rates differed between the two IOLs. In IOL A 14 of 29 (48\%) and in IOL B 26 of 29 (90\%) IOLs developed regeneratory after cataract. The pearl subtype was seen in $9(31 \%)$ IOL A eyes and $12(41 \%)$ IOL B eyes. The monolayer subtype occurred in $5(17 \%)$ and 12 eyes (41\%), respectively. Syncytium occurred in 4 (14\%) and 12 eyes $(41 \%)$ in each group, respectively.

The IOLs showed statistically significant differences with relation to the strength/degree of anterior fibrosis, IOL A showed no fibrosis in 27 eyes $(69 \%)$ whereas in IOL B in 4 eyes $(11 \%)$ no anterior fibrosis was present $(n=36 ; p<$ 0.01 ; McNemar).

The strength of anterior fibrosis did not correlate significantly with the amount of PCO AQUA Score in either of the two groups (IOL A Spearman's $r=0.138, p=0.43$; IOL B Spearman's $r=0.36, p=0.18$ ).

When capsular bend configuration at the optic rim was assessed for each IOL type, the findings showed three main types of capsular bend configuration: parallel (no capsular fusion at the optic rim), Y (loose capsular fusion close to the optic rim) and right angle (close optic/capsule attachment at the optic rim). The IOL A exhibited these configurations at respective rates of $3(7 \%), 6(15 \%)$, and $19(49 \%)$. Eleven (28\%) IOLs could not be evaluated due to insufficient mydriasis. The IOL B group had respective configurations of $5(13 \%), 18(46 \%)$, and $9(23 \%)$. Seven (18\%) IOLs could not be evaluated. Intraindividual differences in PCO occurrence between IOL A and IOL B are shown in Fig. 2 (Fig. 4(supplemental)). A summary of the most relevant observations is shown in Table 2 .

No serious adverse events other than PCO development occurred among the study group.

\section{Discussion}

This study compares the PCO and YAG laser capsulotomy rates of two commonly IOLs: The EyeCee One (IOL A) and iMics1 (IOL B) IOL. Both IOLs are made from a hydrophobic acrylic material and have a single-piece design. Studies have shown that square-edged IOLs produce lower rates of PCO than round-edged IOLs [9-11]. A sharp posterior optic edge inhibits the migration of lens epithelial cells behind the IOL optic [12]. It is thought that not only the presence of a sharp posterior optic edge, but also differences in sharpness play a role in preventing PCO [13].

A previous study by Leydolt et al. also compared two single-piece sharp-edged hydrophobic acrylic IOLs, again one with a biplanar design with a continuous sharp posterior edge (IOL 1: AMO Tecnis ZCB00), and the other with a uniplanar design and a discontinuous edge (IOL 2: Alcon AcrySof SA60AT). In 56 eyes of 23 patients they did not find such a significant difference in PCO after 3 years with objective image analysis. The authors concluded that the potential advantages in IOL design of IOL 1 (posterior optic offset, continuous posterior optic edge) might have been outbalanced by advantageous material properties of IOL 2 [2].

How can the differences in PCO and YAG performance of the EyeCee One (IOL A) and iMics IOLs (IOL B) found in this study be explained? First, although both lenses were hydrophobic, the different material in each lens may differ in their interaction with the lens epithelial cells and the capsule. This view is encouraged by a recent study from our group, comparing two hydrophobic acrylic IOLs of exactly the same design. The Acrysof SA60AT IOL presented impressive lower PCO and Nd:YAG capsulotomy rates compared to the Focus Force AS60125 IOL after 3 years. Differences in the hydrophobic material could be the reason, provided that posterior edge sharpness is comparable with both IOLs. This view is supported by the observation that both lenses showed the same amount and pattern of barrier failure at the optic/haptic junction at the 18 months follow-up examination [14] Second, though both IOLs have a similar design, they do differ in some design criteria: Haptics of IOL A are not angulated while haptics of IOL B are angulated by $5^{\circ}$; IOL A has a slightly larger overall diameter of $13 \mathrm{~mm}$, while IOL B has a slightly smaller diameter of $12.5 \mathrm{~mm}$. Another difference is the biplanar design of IOL A with a posterior optic offset compared to the uniplanar design of IOL B. IOL A has a step between the haptic and optic posterior surface (Fig. 3, Fig. 5 (Supplemental)). With IOL $\mathrm{B}$, the posterior sharp edge is interrupted along the optic-haptic junctions. Also, there are differences in the actual sharpness of the posterior optic edge: A recent study from Nanavaty et al. evaluating the sharpness of the posterior optic edge, applying the same method the study group published in 2008 of measuring the radius of the curvature of the posterior optic edge, showed a significantly sharper posterior optic edge (smaller radius $=$ sharper optic edge) for IOL A with a radius of $8.6 \mu \mathrm{m}$ compared to $19.7 \mu \mathrm{m}$ for IOL B $[15,16]$. The biplanar design of IOL A also explains the difference in the strength of anterior fibrosis between the groups. With the uniplanar IOL $B$, the rhexis leaf readily settles down on the optic surface, while with IOL A, the elevated optic rim withholds the anterior capsule leaf from touching down on the optic. This leads to less fibrotic reaction. This is in accordance to a study by Johansson comparing two IOLs of uni- and biplanar design [17].

What is the possible impact of these differences in design? Angulation and posterior optic offset should stretch out the capsule and enhance contact to the optic and thus reduce $\mathrm{PCO}$ and in particular pearl formation. So should a larger overall diameter, but it may also favour PCO formation by hindering capsular bending along the IOL axis. 
Fig. 2 Examples of observed $\mathrm{PCO}$ in the same patient with different IOLs and different AQUA Score. Epithelial lens cell ingrowth over the optichaptic junction as the main pathway for PCO development was observed in both IOLs

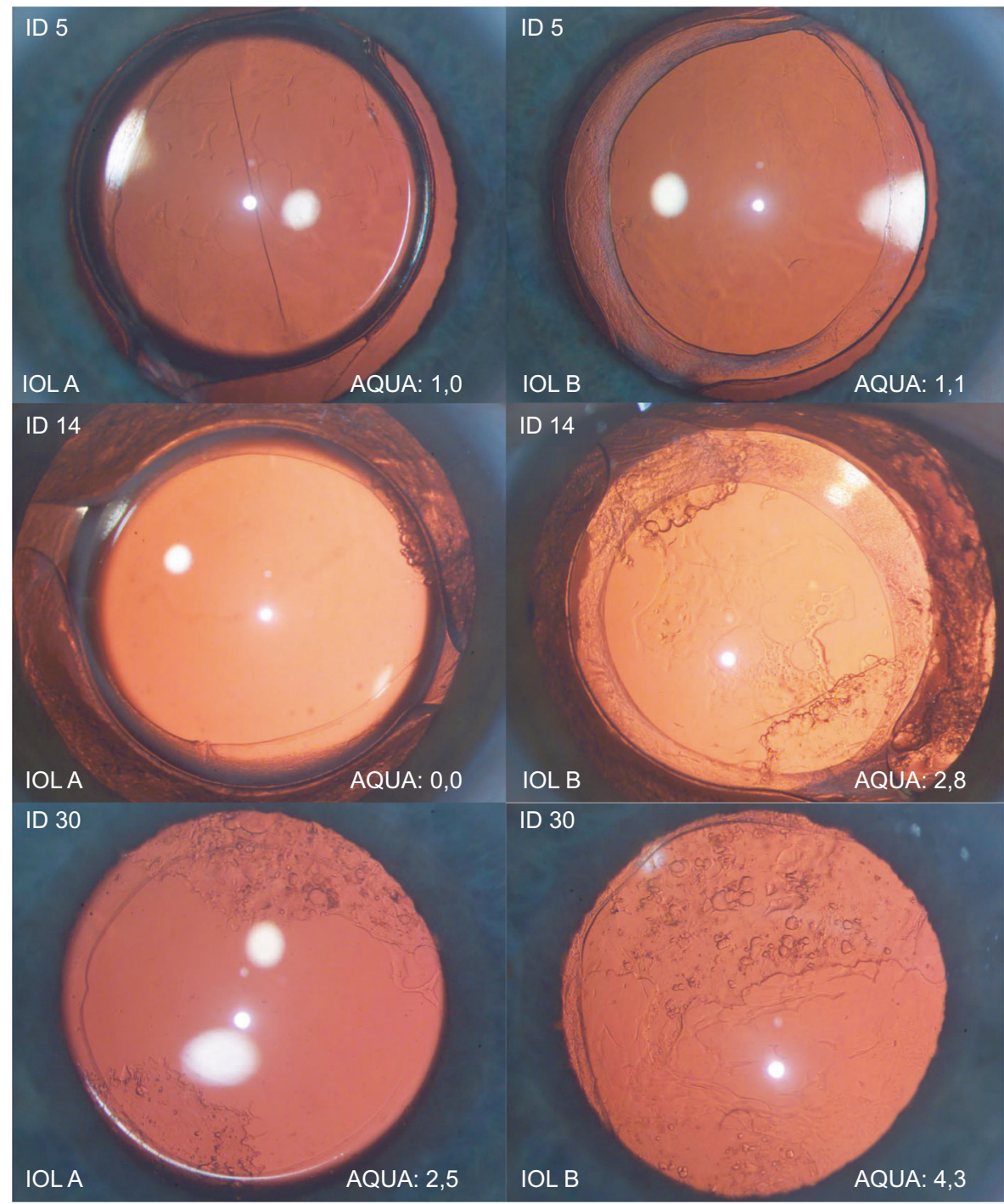

Table 2 Summary of the most relevant observations

\begin{tabular}{llll}
\hline & IOL A & IOL B & Significance \\
\hline AQUA Score $(n=29)$ & $1.57 \pm 1.63$ & $2.45 \pm 1.44$ & $p=0.019$ \\
BCVA (logMAR) $(n=29)$ & $0.032 \pm 0.053$ & $0.089 \pm 0.11$ & $p<0.01$ \\
Nd:YAG after 3a $(n=39)$ & $6(15 \%)$ & $18(46 \%)$ & $p<0.01$ \\
Ant. LC-Gap $(n=37)$ & $33(89 \%)$ & $5(13 \%)$ & $p<0.01$ \\
Post. LC-Gap $(n=29)$ & $22(76 \%)$ & $10(35 \%)$ & $p<0.01$ \\
Ant. Fibrosis $(n=36)$ & $9(25 \%)$ & $32(89 \%)$ & $p<0.01$ \\
\hline
\end{tabular}

$B C V A$ Best Corrected Visual Acuity, Nd-YAG Neodymium:YttriumAluminium-Garnet capsulotomy rate after 3 years Ant. LC-Gap anterior lens-capsule distance, Post. LC-Gap posterior lens-capsule distance, Ant. Fibrosis anterior fibrosis

In our study, IOL A has a slightly bigger overall diameter of $13.0 \mathrm{~mm}$ than IOL B with $12.5 \mathrm{~mm}$. In a recent study Mylonas et al. found that the overall diameter of an IOL did not influence PCO rate. They compared two similar design IOLs with overall diameters of $11 \mathrm{~mm}$ and $10.3-10.8 \mathrm{~mm}$ depending on the dioptric power, respectively. No significant differences in PCO and YAG rates were found after 12 months [18]. However, PCO follow-up time in that study was too short for the results to be meaningful and translated to the current 3-years' study findings. A stepped or "enhanced" edge beneath the haptic-optic junction has been attributed to withhold LEC ingrowth. However, in group A, with 13 out of 14 IOLs with barrier failure LEC ingrowth developed beneath the optic-haptic junctions. Since the junction is narrow, LEC ingrowth in almost all these cases did not reach the optic centre, explaining for the still low YAG capsulotomy rate of $15.4 \%$. The assumption that the stepped or continuous edge explains for a better PCO performance is therefore not substantiated by these findings. Considering the inability of a stepped edge beneath a broad haptic base to allow for capsular bend formation and capsular sealing with the anterior capsule, this is little surprising. 


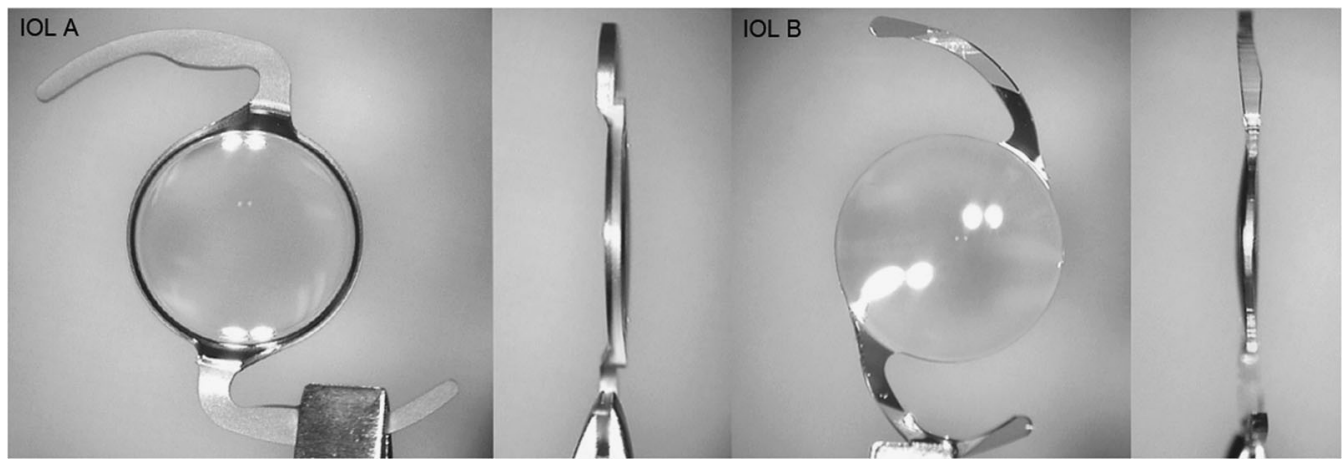

Fig. 3 Axial and profile view of both IOLs taken with the operating microscope: Left IOL A; right IOL B

The greater sharpness of the optic edge of IOL A may well be a factor for the better performance. A previous study of our group compared PCO and capsulotomy rates of the iMics1 (IOL B in the current study) with another singlepiece nonangulated hydrophobic acrylic IOL: the AcrySof SN60WF IOL. Intraindividually 200 eyes were comprised. These two IOLs have a very similar design with no angulation and a discontinuous sharp posterior optic edge. PCO was scored using the same method as in the current study. A mean score of $3.0 \pm 2.0$ was found with the iMics1 and $1.9 \pm 1.4$ with the AcrySof SN60WF IOL $(p<0.01)$ after 3 years. Within 3 years, $35.6 \%$ of the iMics 1 eyes needed Nd: YAG capsulotomy versus $13.7 \%$ of the eyes implanted with an AcrySof SN60WF IOLs. Accordingly, the authors concluded that differences in the hydrophobic acrylic material must have outperformed the greater sharpness of the optic edge [19]. Since the recent edge profile evaluation study by Nanavaty et al. revealed new data on optic edge sharpness, proving a sharper optic edge with the SN60WF IOL vs. the iMics 1 IOL, the results in this comparative study (SN60WF vs. iMics 1) could not only be explained by the favourable hydrophobic material as the authors mentioned, but also with the much sharper posterior optic edge $[15,16]$. The PCO score of 3.0 reported for the iMics IOL in the study just quoted were similar to that of 2.45 observed with the iMics1 in the current study. This is also true for the YAG capsulotomy rates of $35.6 \%$ and $46.2 \%$, respectively. This corroborates the consistency of our scoring methods and YAG laser indications.

A tight contact of the anterior and posterior capsule with the optic should reduce PCO rates by enhancing capsular sealing along the optic rim and by reducing the space for retro-optical pearl formation [20]. The current study found lower PCO rates with IOL A, exhibiting an anterior and posterior optic-capsule interspace (gaping) in $89 \%$ and $76 \%$, respectively, compared to IOL B that has respective rates of just $13 \%$ and $24 \%$. The higher percentage of separation between the anterior capsule and optic surface found with IOL A is explained by its posterior optic offset, which should provide tighter contact between optic and anterior capsule. However, separation between the posterior capsule and optic was also significantly more frequent with IOL $\mathrm{A}$ in spite of its biplanar design. This finding is unexpected and contradicts Nixon et al. who concluded that the biplanar hapticoffset design of an IOL would bring about a tight posterior optic-capsule contact [3]. The significantly lower PCO and YAG rates in spite of the more frequent occurrence of opticcapsule space between the anterior and posterior lens capsule and IOL optic can only be explained by the dominating impact of a sharper posterior optic edge and differences in material properties. No significant adverse events were observed among the study participants throughout the 3-year follow-up period, suggesting both IOLs are safe for use in cataract surgery patients.

The main limitation of this study is the high dropout rate of 41 patients, which is not unusual in this elderly study population. Furthermore, an additional follow-up visit after 1.5 years could have encouraged patients' compliance for long-term follow-ups. Additionally, 1.5-year follow-up and AQUA PCO data would allow to include drop-outs due to incurrent Nd:YAG capsulotomy by extrapolation, according to a statistical model by Buehl et al. [21]. To allow for this, future studies investigating long-term intraindividual PCO performance should include an intermediate follow-up visit. Still, the results in this study showed clear and statistically significant differences between the IOLs investigated.

In conclusion, of the two hydrophobic IOLs compared, IOL A with its biplanar design and its sharper and continuous posterior optic edge showed significantly lower PCO and YAG rates than IOL B with its less sharp and discontinuous edge. The study shows that sharpness of the edge and differences in the hydrophobic material itself may be more important than posterior angulation or a posterior optic offset. The particular pattern of barrier failure and PCO development starting out from the haptic junctions of IOL A allow the conclusion that a stepped sharp optic edge beneath a haptic junction will not be effective in withholding LEC migration in the long run. 


\section{Summary}

\section{What was known before}

- A sharp posterior optic edge inhibits lens epithelial cell migration along the posterior capsule.

- The optic/haptic junctions are the preferred pathway for PCO ingrowth through the optic centre.

\section{What this study adds}

- PCO development was significantly lower with the EyeCee One vs. the iMics 1 IOL.

- The 360 degree posterior optic edge seems to be ineffective beneath the optic/haptic junctions.

- A tight posterior capsule/optic fitting plays a minor role in PCO development.

\section{Compliance with ethical standards}

Conflict of interest The authors declare that they have no conflict of interest.

Publisher's note: Springer Nature remains neutral with regard to jurisdictional claims in published maps and institutional affiliations.

\section{References}

1. Leydolt C, Schartmüller D, Schwarzenbacher L, Schranz M, Schriefl S, Menapace R. Comparison of posterior capsule opacification development with 2 single-piece intraocular lens types. J Cataract Refract Surg. 2017;43:774-80. https://doi.org/10.1016/j. jcrs.2017.06.005

2. Leydolt C, Kriechbaum K, Schriefl S, Pachala M, Menapace R. Posterior capsule opacification and neodymium:YAG rates with 2 single-piece hydrophobic acrylic intraocular lenses: three-year results. J Cataract Refract Surg. 2013;39:1886-92.

3. Nixon DR, Woodcock MG. Pattern of posterior capsule opacification models 2 years postoperatively with 2 single-piece acrylic intraocular lenses. J Cataract Refract Surg. 2010;36:929-34. https://doi.org/10.1016/j.jcrs.2009.12.040

4. Menapace R, Wirtitsch M, Findl O, Buehl W, Kriechbaum K, Sacu S. Effect of anterior capsule polishing on posterior capsule opacification and neodymium:YAG capsulotomy rates: three-year randomized trial. J Cataract Refract Surg. 2005;31:2067-75. https://doi.org/10.1016/j.jcrs.2005.08.051

5. Buehl W, Findl O, Menapace R, Georgopoulos M, Rainer G, Wirtitsch $\mathrm{M}$, et al. Reproducibility of standardized retroillumination photography for quantification of posterior capsule opacification. J Cataract Refract Surg. 2002;28:265-70.

6. Findl O, Buehl W, Menapace R, Georgopoulos M, Rainer G, Siegl H, et al. Comparison of 4 methods for quantifying posterior capsule opacification. J Cataract Refract Surg. 2003; 29:106-11.
7. Findl O, Buehl W, Siegl H, Prinz A. Removal of reflections in the photographic assessment of PCO by fusion of digital retroillumination images. Invest Ophthalmol Vis Sci. 2003;44:275-80.

8. Sacu S, Findl O, Menapace R, Buehl W. Influence of optic edge design, optic material, and haptic design on capsular bend configuration. J Cataract Refract Surg. 2005;31:1888-94. https://doi. org/10.1016/j.jcrs.2005.03.072

9. Buehl W, Findl O, Menapace R, Rainer G, Sacu S, Kiss B, et al. Effect of an acrylic intraocular lens with a sharp posterior optic edge on posterior capsule opacification. J Cataract Refract Surg. 2002;28:1105-11.

10. Buehl W, Findl O, Menapace R, Sacu S, Kriechbaum K, Koeppl C, et al. Long-term effect of optic edge design in an acrylic intraocular lens on posterior capsule opacification. J Cataract Refract Surg. 2005;31:954-61. https://doi.org/10.1016/j.jcrs.2004.09.053

11. Sacu S, Menapace R, Findl O, Kiss B, Buehl W, Georgopoulos M. Long-term efficacy of adding a sharp posterior optic edge to a three-piece silicone intraocular lens on capsule opacification: fiveyear results of a randomized study. Am J Ophthalmol. 2005;139:696-703. https://doi.org/10.1016/j.ajo.2004.12.050

12. Nishi O, Nishi K, Mano C, Ichihara M, Honda T. The inhibition of lens epithelial cell migration by a discontinuous capsular bend created by a band-shaped circular loop or a capsule-bending ring. Ophthalmic Surg Lasers. 1998;29:119-25.

13. Werner L, Tetz M, Feldmann I, Brücker M. Evaluating and defining the sharpness of intraocular lenses: microedge structure of commercially available square-edged hydrophilic intraocular lenses. J Cataract Refract Surg. 2009;35:556-66. https://doi.org/ 10.1016/j.jcrs.2008.11.042

14. Schartmüller D, Schriefl S, Schwarzenbacher L, Leydolt C, Menapace R. Posterior capsule opacification of two hydrophobic, acrylic intraocular lenses: 3 year follow up. 36th Congress of the ESCRS. Vienna, 2018.

15. Nanavaty MA, Spalton DJ, Boyce J, Brain A, Marshall J. Edge profile of commercially available square-edged intraocular lenses. J Cataract Refract Surg. 2008;34:677-86. https://doi.org/10.1016/ j.jcrs.2007.12.024

16. Nanavaty MA, Zukaite I, Salvage J Edge profile of commercially available square-edged Intraocular lenses: Part 2. J Cataract Refract Surg. 2019;45:847-53

17. Johansson B. Glistenings, anterior/posterior capsular opacification and incidence of $\mathrm{Nd}$ :YAG laser treatments with two aspheric hydrophobic acrylic intraocular lenses - a long-term intra-individual study. Acta Ophthalmol. 2017;95:671-77. https://doi.org/10. 1111 /aos. 13444

18. Mylonas G, Georgopoulos M, Prinz A, Vock L, Blum RA, Schmidt-Erfurth U. Influence of a variable overall diameter hydrophilic acrylic sharp-edged single-piece intra-ocular lens on capsule opacification one year after surgery. Curr Eye Res. 2014;39:620-5. https://doi.org/10.3109/02713683.2013.863939

19. Leydolt C, Schriefl S, Stifter E, Haszcz A, Menapace R. Posterior capsule opacification with the iMics1 NY-60 and AcrySof SN60WF 1-piece hydrophobic acrylic intraocular lenses: 3-year results of a randomized trial. Am J Ophthalmol. 2013;156:375-81 e2. https://doi.org/10.1016/j.ajo.2013.04.007

20. Menapace R. Prevention of posterior capsule opacification. New York, NY: Springer; 2005. p. 101-22.

21. Buehl W, Heinzl H, Mittlboeck M, Findl O. Statistical problems caused by missing data resulting from neodymium:YAG laser capsulotomies in long-term posterior capsule opacification studies: problem identification and possible solutions. J Cataract Refract Surg. 2008;34:268-73. https://doi.org/10.1016/j.jcrs.2007.11.014 